\title{
Transdermal Route of Administration
}

National Cancer Institute

\section{Source}

National Cancer Institute. Transdermal Route of Administration. NCI Thesaurus. Code C38305.

Administration of a substance through the dermal layer of the skin into the systemic circulation by diffusion. 